\title{
Devastación y catástrofe en el arte contemporáneo: tácticas latinoamericanas de destrucción constructiva
}

\author{
Jazmín Adler \\ Consejo Nacional de Investigaciones Científicas y Técnicas (CONICET) \\ Universidad Nacional de Tres de Febrero (Buenos Aires, Argentina)
}

RESUMEN:

El presente artículo analiza el papel desempeñado por la destrucción y la catástrofe en el arte contemporáneo, particularmente en torno a aquellas prácticas artísticas que se valen de medios y herramientas tecnológicos para desencadenar procesos de devastación, acumulación de vestigios e investigaciones con diversas materialidades efímeras. Luego de sondear algunos antecedentes fundamentales sobre la atracción ejercida por el acto auto-destructivo en el campo artístico durante las últimas décadas, el trabajo estudia un conjunto de obras latinoamericanas que encarnan tácticas de destrucción constructiva. Los vestigios producidos por estas obras no remiten a un pasado perdido, sino que el propio acto de devastación en Latinoamérica supone una operación estético-política de transformación y resistencia crítica, dirigida a cuestionar las estructuras dominantes.

PALABRAS CLAVE:

arte contemporáneo, catástrofe, destrucción, Latinoamérica, tecnología.

\section{ABSTRACT:}

This article addresses the role played by destruction and catastrophe within contemporary art, especially in practices that intersect art and technologies to display processes of devastation, accumulation of vestiges and investigations with ephemeral materials. After studying some artworks that have explored auto-destructive act as an artistic gesture throughout the last decades, the article analyses certain Latin-American projects that embody tactics of constructive destruction. The ruins produced by these works do not refer to the past. Instead, the act of devastation in the region could be interpreted as an aesthetic and political strategy of critical resistance towards the status quo.

KEYWORDS:

contemporary art - catastrohpe - destruction - Latin-America - technology. 


\section{Arte auto-destructivo}

En su primer Manifiesto, fechado en noviembre de 1959, Gustav Metzger propuso la noción de arte auto-destructivo para referir a diferentes prácticas artísticas que encarnaban expresiones de arte público en sociedades industriales ${ }^{1}$. Metzger aludía a pinturas, esculturas e instalaciones creadas a partir de fuerzas naturales, técnicas tradicionales e inclusive medios tecnológicos, las cuales podían ostentar una vida útil de entre un momento breve y veinte años ${ }^{2}$. Estos proyectos delineaban una nueva concepción de obra, en cuyas bases la sacralidad del objeto físico, estático y perdurable era reemplazada por la transitoriedad del acto de la destrucción, así como por el carácter efímero de sus consecuencias: los vestigios.

El texto del cual provienen las ideas precedentes acompañaba a la primera muestra del artista, organizada en un café ubicado en el número catorce de la calle Monmouth Street de la ciudad de Londres. Allí Metzger expuso un conjunto de cartones extraídos del embalaje de un televisor. Desarmados en diferentes partes, los fragmentos de la caja eran exhibidos colgando de una pared, en una operación que ha sido interpretada a modo de cita tanto de los ready-mades duchampianos, como de los relieves realizados por artistas del constructivismo ruso $^{3}$. Las propuestas estéticas que Metzger desarrollaría desde entonces, y durante la década siguiente en el contexto de la Guerra Fría, se inscribieron en un programa de protesta radical contra las armas nucleares, la expansión del capitalismo y las lógicas consumistas de un mercado del arte que históricamente había perseguido la producción de objetos materiales sustentados en la noción de valor. Ante estas dinámicas mercantiles, el arte auto-destructivo valorizaba procesos de deterioro y se aseguraba de no dejar ningún resto físico ni asible de la destrucción. En palabras de Metzger: "Cuando el proceso de desintegración se completa, la obra es removida del sitio y desguazada".

METZGER, Gustav, Auto-Destructive Art (Manifesto) (Manuscrito), Londres, 1959, s/p. Disponible en: https:// monoskop.org/Gustav_Metzger [consulta: 15-11-19].

METZGER, Gustav, Auto-Destructive..., opus cit., s/p.

WILSON, Andrew, "Gustav Metzger's Auto-Destructive/Auto-Creative Art. An Art of Manifesto, 19591969”, en Third Text, 22, 2, Londres, Routledge, 2008, p. 182.

4 METZGER, Gustav, Auto-Destructive..., opus cit., s/p.
Sin embargo, el acto destructivo del objeto era pensado en conexión estrecha con el concepto de creación. Las ideas de Metzger alcanzaron un punto culminante en un evento difundido como The Destruction in Art Symposium (DIAS), organizado por el artista en 1966 y desarrollado en el Africa Centre, en Covent Garden, Londres. El simposio apuntaba a reunir artistas, escritores, sociólogos, psicólogos y otros especialistas, con el objetivo de discurrir sobre el fenómeno de auto-destrucción en el arte y en la sociedad contemporánea en su conjunto. La historiadora del arte Kristine Stiles ha sugerido que la destrucción "en" arte no es sinónimo de la destrucción “del arte”, debido a que la primera actitud introduce el aspecto destructivo en el quehacer artístico, a su vez que discurre sobre el papel de la destrucción no solo en el proceso creativo, sino también en la vida en general ${ }^{5}$. Según Stiles, los ejes del debate promovido por DIAS ahondaban en el cataclismo de los materiales, el destrozo de las obras ocasionado por la naturaleza, y las agresiones psicológicas y físicas generadas sobre el cuerpo ${ }^{6}$.

Asimismo, DIAS dio cuenta de una progresiva inclinación de Metzger hacia el estudio de metodologías y procedimientos de la ciencia y la tecnología: biología, economía, medicina, física y astronomía son algunos de los campos disciplinares mencionados por el artista en el evento ${ }^{7}$. Ya unos años atrás, en el artículo $A u$ to-Destructive Art Machine Art Auto-Creative Art, su autor declaraba:

Auto-destructive art and auto creative art aim at the integration of art with the advances of science and technology. The immediate objective is the creation, with the aid of computers, of works of art whose movements are programmed and include "self-regulation".

STILES, Kristine, "Synopsis of The Destruction in Art Symposium (DIAS) and Its Theoretical Significance", en The Act, 1, 2, 1987, p. 22.

6 Participaron Günter Brus, Ivor Davies, Pro Diaz, Al Hansen, Fred Hunter, Gustav Metzger, Otto Mühl, Yoko Ono, Ralph Ortiz, Robin Page, Jasia Reichardt, Werner Schreib, John Sharkey, Hanns Sohm, Biff Stevens y Wolf Vostell.

${ }^{7}$ El interés creciente de Metzger hacia la ciencia y la tecnología han sido detenidamente estudiados por Andrew Wilson, en el trabajo citado en la nota tres. Entre los antecedentes de este interés, Wilson destaca la investigación de cristales líquidos, desarrollada por el artista entre 1965 y 1966. 
The spectator, by means of electronic devices can have a direct bearing on the action of these works. ${ }^{8}$

La relación entre los procesos artísticos de destrucción/creación en vinculación con la ciencia y la tecnología estaba siendo investigada por Jean Tinguely hacia la misma época. Su obra Hommage à New York, presentada en el jardín de esculturas del Museo de Arte Moderno de Nueva York, a comienzos de 1960, precisamente había surgido de la colaboración con el ingeniero Billy Klüver, quien trabajaba en los Laboratorios Bell ${ }^{9}$. Ubicada en una frontera difusa entre el happening y la performance, la obra era definida por Tinguely como situación ${ }^{10}$ y fue diseñada como una gran máquina efímera, integrada por ruedas, motores, bocinas, líquidos químicos, radios, una máquina de escribir, bocinas y un piano. Los múltiples elementos y componentes estaban dispuestos de tal manera que, al cabo de veintisiete minutos, la maquinaria se autodestruyó por completo ${ }^{11}$. El artista había concebido a Hommage à New York como un proyecto que socavaba la posibilidad de "museificar" el objeto-obra y proponía, en cambio, una experiencia momentánea:

Una obra efímera, pasajera como una estrella fugaz $y$, sobre todo, destinada a no ser recuperada por los museos. Ella no debería ser museificada. Era necesario que ocurriese, que se la soñara y que se hablara de ella, y eso es todo; al día siguiente ya no debería haber nada más. Todo retornaría a la basura. Ella poseía una cierta sofistica-

8 METZGER, Gustav, "Machine, Auto-Creative and Auto-Destructive Art", en Ark. Journal of the Royal College of Art, 32, Londres, 1962, pp. 7-8.

$9 \quad$ En 1966, Billy Klüver fundaría la organización E.A.T (Experiments in Art and Technology) junto a Robert Rauschenberg, Fred Waldhauer y Robert Whitman, una asociación sin fines de lucro destinada a propiciar intercambios entre artistas e ingenieros, quienes a partir de entonces trabajaron en conjunto en el desarrollo de obras artístico-tecnológicas.

10 El término "situación" para caracterizar a la obra es planteado por el propio Tinguely en el video de registro de Hommage à New York. Disponible en: https://www. youtube.com/watch?v=0MqsWqBX4wQ [consulta: 1611-19].

11 Por ejemplo, algunas zonas metálicas de la máquina fueron derretidas a causa de una resistencia que era sobrecalentada, un globo meteorológico estaba dispuesto para ser explotado, y un balde de gasolina fue vertido sobre un conjunto de velas colocadas sobre el teclado de un piano. ción complicada que la destinaba a la autodestrucción; era una máquina que se suicidaba. ${ }^{12}$

\section{Sobre la destrucción en el arte argentino}

El contexto del arte latinoamericano de los años sesenta también se veía convocado por la potencia del acto destructivo. En noviembre de 1961, la Galería Lirolay de Buenos Aires organizaba la exposición Arte destructivo, un evento sin antecedentes en el ámbito local, en el cual se exhibieron obras de Kenneth Kemble, Enrique Barilari, Olga López, Jorge López Anaya, Jorge Roiger, Antonio Seguí, Silvia Torras y Luis Wells. Emplazados en distintos puntos de la sala, un conjunto de objetos diversos evocaban las ideas de destrucción y desastre, como si se tratara de piezas devastadas a causa de algún accidente o catástrofe: paraguas destruidos, muñecas amputadas, un sillón tajeado de cuyo interior brotaba la lana del relleno, ataúdes desgastados (uno de ellos baleado), una bañadera manchada con pintura, botellas quebradas, restos de barcos y otras piezas deterioradas, por ejemplo obras informalistas destruidas. Todas estas piezas eran acompañadas por experiencias sonoras, como un discurso de Jorge Romero Brest, piezas musicales creadas por los aristas y fragmentos de Poética de Aristóteles fusionados con partes de la pieza teatral de Picasso conocida como $\mathrm{El}$ deseo atrapado por la cola, entre otras. Azorada por la estetización del destrozo, la crítica describió al evento como "un basural artísticamente montado", "un arpilleresco, sucio, despanzurrado y lastimoso reducto" y "una dársena pestilente de lo vacío y lo putrefacto"13. De esta manera, la destrucción encarnaba diversas formas y se materializaba a través de medios, soportes y formatos que expandían los límites del arte conocido. Algunas piezas realizaban un acto de destrucción física mediante balas, fuego, cortes o martillazos; otras investigaban la devastación provocada por fuerzas físicas o naturales a lo

\footnotetext{
12 TINGUELY, Jean, “Tinguely parle de Tinguely”, emisión de la Radio-Televisión belga de la comunidad francesa presentada por Jean-Pierre Van Tieghem, 13 diciembre de 1982.

13 LONGONI, Ana, "Llamen a Manliba. Dos críticas de los primeros ‘60 arremeten contra la vanguardia”, en Ramona. Revista de Artes Visuales, 15, Buenos Aires, 2001, p. 30.
} 
largo del tiempo; ciertos proyectos recurrían a la destrucción verbal alterando las lógicas imperantes en textos preexistentes ${ }^{14}$; $\mathrm{y}$ un conjunto de trabajos alteraban la música tradicional investigando la posibilidad creativa ofrecida por el ruido y la distorsión del sonido.

Reconociendo el desconcierto que una muestra semejante podía llegar a generar entre sus visitantes, Kenneth Kemble explicaba que la exposición tenía un "carácter de experimento, de un ensayo tentativo", cuya intención era aventurarse a lo desconocido y dejar un camino abierto para experiencias ulteriores en torno a la exploración de la destrucción. Al igual que Metzger, Kemble argüía que el arte no podía permanecer ajeno ante la magnitud y consecuencias de la bomba atómica: “¿Es que se puede pretender que el artista, que siempre ha actuado como antena receptora de lo por venir, permanezca insensible ante tal eventualidad?"15. Sin embargo, en un contexto como el argentino, profundamente influido por el psicoanálisis, la destrucción era pensada como una vía factible para canalizar la tendencia destructiva innata del sujeto, frecuentemente atraído por la posibilidad de romper, incendiar, golpear o descomponer toda clase de objetos y materiales, guiado por una "dosis de sadismo o masoquismo más o menos desarrollados [que todos tenemos] pero que no queremos admitir" ${ }^{16}$.

Si bien no es seguro que Kemble haya conocido el trabajo de Metzger ${ }^{17}$-de hecho, en el texto previamente citado, el artista argentino sostiene que el tema nunca hasta ese momento había sido "investigado a fondo"18 -, lo cier-

14 Este tipo de operación fue realizada por Kenneth Kemble en Maqueta para un monumento a Lamuel Lumija Mainez. Un cajón colgado de uno de los muros presentaba en su interior una antorcha empapada con petróleo. Cubierto por una tela blanca, el conjunto proyectaba una sombra sobre la pared simulando un perfil humano. El título de la obra propone un juego de palabras a partir del nombre Manuel Mujica Láinez (1936-1984), reconocido escritor y periodista argentino, quien se desempeñó como crítico de arte en el diario La Nación.

15 KEMBLE, Kenneth, Arte destructivo, en Arte destructivo: Barilari, Kemble, López Anaya, Roiger, Seguí, Torrás, Wells, Buenos Aires, Galería Lirolay, exp. 20 al 30 de noviembre de 1961, s/p.

16 KEMBLE, Kenneth, Arte destructivo, opus cit., s/p.

17 LÓPEZ DE SILVA CARLOS, Félix, "Estética y creación en torno a la destrucción. El caso del arte destructivo", en Activarte. Revista Independiente de Arte, Teoria de las Artes, Pedagogía y Nuevas Tecnologías, Sevilla, 4, 2011, pp. 59-69.

18 KEMBLE, Kenneth, Arte destructivo, opus cit., s/p. to es que la conexión entre el arte destructivo en Argentina e Inglaterra llegó de la mano de Luis Wells, en 1966, cuando el artista viajó a Londres con una beca otorgada por el British Council. En su estadía londinense, Wells decidió inscribirse en DIAS. De acuerdo con la investigación de Stiles ${ }^{19}$, el artista argentino presentó en el simposio documentación sobre la muestra de la galería Lirolay. Fotografías, textos y audios permitieron difundir la experiencia en el ámbito europeo, y compartir algunas ideas en torno a las estrategias implementadas por la exposición sudamericana para jaquear la ortografía, la semántica y el sonido vocalizado.

\section{Ruinas en (y del) territorio en la confluencia arte/tecnología}

La investigación material, estética y poética acerca del carácter creativo de la destrucción ha sido frecuente en diversas expresiones del arte contemporáneo. Las prácticas examinadas en las páginas precedentes demuestran que, desde mediados del siglo pasado, algunos artistas vieron en la acción destructiva una vía eficaz para cuestionar la histórica primacía de la obra objetual, y ahondaron en el carácter constructivo que habita en todo gesto de destrucción. Además de los proyectos previamente estudiados, así lo demuestran otra propuestas coetáneas a aquellos, como los building cuts de Gordon Matta-Clark, quien se concebía a sí mismo como un "arqueólogo urbano" ${ }^{20}$, en cuyos cortes afloraba la memoria contenida en los edificios ${ }^{21}$; la riqueza de los escombros explorada por Robert Smithson ${ }^{22}$, un artista cautivado por las ruinas industriales de New Jersey de los años sesenta, "monumentos del paisaje posindustrial trastornado por la modernización urbana" ${ }^{23}$; o bien las reconfiguraciones y reutilizaciones de objetos variados, provenientes

19 STILES, Kristine, "The Story of the Destruction in Art Symposium and the DIAS affect", en Gustav Metzger: History History, Viena, Ostfildern-Ruit, 2005, pp. 41-65.

20 DONOSO, Pedro (ed.), Gordon Matta-Clark: Experience Becomes the Object, Barcelona, Poligrafa, 2016, p. 114.

21 RUSSI KIRSCHNER, Judith, “Non-uments”, en Gordon Matta-Clark, Valencia, IVAM - Institut Valencià d' Art Modern, exp. 3 de diciembre de 1992 al 31 de enero de 1993.

22 SMITSHON, Robert, "The Monuments of Passaic", en Artforum, 6, 4, 1967, pp. 48-51.

23 SPERANZA, Graciela, Cronografias: Arte y ficciones de un tiempo sin tiempo, Barcelona: Anagrama, 2017, p. 28. 
de contextos y tiempos diversos, desarrolladas por Sonia Andrade en una obra como Hydragrammas (1978-1993). En este último caso, la colección de vestigios de objetos del pasado es convertida en un conjunto de piezas únicas que transforman "fragmentos del mundo en minúsculas piezas de existencia" 24 .

En la contemporaneidad más reciente, resulta frecuente hallar propuestas que renuevan la reflexión acerca del binomio destrucción/ creación y la recuperación de vestigios, a través de la elaboración de archivos, el acto de la devastación, la acumulación de sus restos y la exploración con materialidades efímeras. Más aun, determinadas obras realizadas en los últimos años abordan la acción catastrófica y la figura de la ruina desde el cruce entre el arte y las tecnologías electrónicas y/o digitales.

La obra de Juan Sorrentino constituye un caso paradigmático en esta dirección. En Derrumbe (2016-2019), el artista argentino indagó en los efectos producidos por un sonido de cuarenta y siete hercios emitido por parlantes contenidos en un gran muro. El revoque de la pared era desprendido y convertido en escombros como consecuencia de la vibración de baja frecuencia. Proyectos posteriores, entre ellos En el monte nunca se está solo (2017), continuaron con la misma línea de investigación. Allí Sorrentino trasgredió los límites del espacio de exhibición convencional a fines de realizar una serie de intervenciones en el paisaje, mediante instalaciones y objetos sonoros emplazados en un terreno de cuatro hectáreas ubicado en el monte chaqueño, en el noreste de Argentina, y librados a los efectos causados por el paso del tiempo. Apelando a las estrategias de derivas y recorridos impulsadas por diversos artistas del Situacionismo y el Land Art, como Richard Long o Hamish Fulton, Sorrentino abrió un sendero a lo largo del cual posteriormente determinaría cinco puntos de escucha. En una etapa ulterior, distribuyó instalaciones sonoras y mecánicas a través del camino, las cuales daban cuenta de un balance del espectro sonoro audible, con sonidos que iban desde el ruido blanco hasta otros sonidos más agudos e inarmónicos. Entre las obras presentadas se encontraba Space

24 ABREU, Hortência, "Sonia Andrade", en VOLZ, Jochen y REBOUÇAS, Julia (eds.), 32nd Bienal de São Paulo: Incerteza Viva, San Pablo Brasil, Fundación Bienal de São Paulo, 2016, p. 350
Scanner, integrada por un gran cono metálico dispuesto sobre una balsa que giraba sobre su propio eje. Mientras la pieza flotaba en el río, emitía un ruido blanco que impactaba sobre distintas superficies como si las fuera escaneando, imprimiendo sobre ellas las características del entorno. En otro de los puntos de escucha se situaba Desmonte, donde un malacate levantaba un conjunto de troncos y posteriormente volvía a emplazarlos sobre la tierra en un ciclo constante, desarrollado al compas del sonido realizado por el dispositivo. Por su parte, en Cenizas de un árbol, un parlante exhibido en el suelo sobre un montículo de cenizas producía un sonido de baja frecuencia; el temblor resultante provocaba que los restos del árbol quemado se disiparan en el aire, sumergiéndolos en una bruma que se esparcía a través del entorno. En la siguiente etapa del proyecto, iniciada una vez que terminaron las acciones, Sorrentino dejó abandonadas las instalaciones de manera que las crecientes del río y las dinámicas del monte fueran transformando a las piezas en ruinas ${ }^{25}$.

La obra de Gabriela Munguía, artista mexicana radicada en Buenos Aires, investiga asimismo la creación sonora a partir de materiales provenientes de la naturaleza y su alteración en vestigios. En el proyecto Máquinas de lo invisible, iniciado en 2017, Munguía se valió de restos del paisaje para desarrollar un site specific que vuelve visible aquellos aspectos naturales que suelen permanecer invisibilizados, percutiendo materiales que, a diferencia de los componentes de las obras de Sorrentino, son externos a la propia naturaleza. Así realizó una serie de acupunturas terrestres, mediante la construcción de máquinas sonoras y lumínicas que captan, procesan y transforman diferentes datos provenientes del entorno natural donde se sitúan, como el electromagnetismo o la radiación solar, fenómenos que no tienden a vislumbrarse en la percepción cotidiana. En la obra producida en el municipio

25 Recientemente Sorrentino creó Monte / Residencia de arte, una iniciativa orientada hacia el arte sonoro y el Land Art. El proyecto consiste en convocar a artistas de diferentes disciplinas para que durante su estadía en el monte creen piezas para el mismo terreno donde tuvo lugar En el monte nunca se está solo. Una vez realizadas, las obras son entregadas al paisaje y así los avatares de la naturaleza convierten a los objetos en vestigios. Un aspecto significativo de la residencia es que ésta involucra una investigación específica sobre aquellos materiales idóneos para devenir en ruinas. 
brasileño de Altamira, durante la Residencia de Arte MUTUCA, Munguía creó una antena meteorológica que captaba diferentes datos recabados de la naturaleza, como la velocidad del viento, la presión atmosférica, la temperatura y la humedad. El proyecto fue titulado Resiliencia \# $1^{26}$ : sonidos del viento. Como si entrañaran vestigios de comportamientos naturales sucedidos de manera permanente en un pasado reciente, dichos restos fueron convertidos por la artista en nuevos materiales para posteriores acciones en el territorio. Una de ellas consistió en la invención de un objeto mecánico sonoro, concebido como una pequeña caja musical, que reproducía la información previamente recopilada y codificada en perforaciones operadas sobre papel. En el caso de la segunda versión del trabajo, Resiliencia \#2: Conversaciones geomorfológicas, realizado en la comuna suiza de Tenna, las piedras del lugar colisionaban con platos de bronce a partir de la fuerza del viento, permitiendo materializar visual y sonoramente los procesos de erosión característicos del territorio intervenido. Esta instalación y performance sonoro-lumínica formó parte de la Bienal Safiental de Land Art, en 2018.

El territorio es también uno de los ejes de la obra de Marcela Armas. La investigación interdisciplinaria de la artista mexicana frecuentemente indaga fenómenos sociales, políticos y económicos, como la delimitación de las fronteras territoriales, la construcción de los relatos históricos, los devenires de la educación en Latinoamérica y la producción energética. Este último tema ha sido el foco de la instalación I-Machinarius (2008), una obra que inquirió las consecuencias de la dependencia energética mexicana en el contexto globalizado. El proyecto fue realizado con motivo de la exposición Sinergia, cuada por Karla Jasso en el Laboratorio de Arte Alameda de la Ciudad de México. La propuesta resultó de un proceso de investigación y discusión desarrollado por un grupo de historiadores, politólogos, especialistas en petróleo y artistas en el momento en que el gobierno de Felipe Calderón iniciaba el proceso de privatización de la industria energética en México. Algunos de los expertos reflexionaron

26 La noción de resiliencia designa la capacidad de adaptación de los seres humanos a toda clase de situaciones hostiles o desfavorables acontecidas en su medio. Los dispositivos, acciones e intervenciones en la naturaleza ideados por Gabriela Munguía actúan como tecnologías sensiblemente humanizadas para propiciar la cooperación entre lo humano y lo no-humano. teóricamente sobre una idea que en ese entonces comenzaba a circular en el ámbito mexicano, de acuerdo a la cual México no contaba con la infraestructura requerida para que las empresas petroleras pudieran continuar en manos del Estado. Por su parte, los artistas realizaron las obras que posteriormente fueron exhibidas en la muestra Sinergia. La propuesta de Armas estuvo integrada por una maquinaria montada sobre la pared de la sala de exposición, compuesta por una cadena industrial, un conjunto de catarinas y un motor que permitía que la estructura estuviera en constante movimiento, mientras chorreaba petróleo crudo sobre el muro. La cadena delineaba el mapa de México invertido, de manera que el petróleo fluía hacia los Estados Unidos. Paulatinamente, la pared blanca iba siendo íntegramente manchada. Aquellos trazos oscuros evocaban la imagen de heridas ensangrentadas, las cuales parecían hacer eco de la metáfora de Eduardo Galeano en Las venas abiertas de América Latina (1971). De manera semejante a los restos producidos por el sonido y la acción de la naturaleza en la obra de Sorrentino, y en sintonía también con los vestigios del paisaje que protagonizan el trabajo de Munguía, en el proyecto de Armas las manchas de petróleo permiten recomponer acciones pasadas a través de sus efectos ruinosos. De acuerdo con la artista, el desbordamiento que propone I-Machinarius convierte al dispositivo en una máquina herida, en tanto alegoría de la "sociedad perturbada por una arraigada idea de incapacidad, fracaso e inseguridad"27. La noción de máquina frágil y vulnerable reaparece en otras obras contemporáneas, en las cuales la acción destructiva también supone una estrategia destinada a cuestionar las estructuras sociales, políticas y culturales.

\section{Máquinas falibles}

La artista brasileña Gabriela Mureb construye objetos y diseña instalaciones que tornan diversos materiales en escombros. En Máquina \#4: piedra (2011-2017), una máquina de aspecto industrial accionada por un motor golpea repetidamente una piedra que se encuentra frente a ella, como si intentara perforarla. Cada uno de los golpes de la máquina contra el bloque hacen que éste pierda el equi-

\footnotetext{
7 ARMAS, Marcela, "I-Machinarius", s/f. Disponible en: https://www.marcelaarmas.net/?works=i-maquinarius [consulta: 18-11-19].
} 
libro y vuelva a recuperarlo en un gesto reiterado carente de sentido. Si bien la aparente improductividad del dispositivo recuerda las operaciones de Tinguely en el desarrollo de sus máquinas inútiles, en las obras de Mureb la ineficacia de la labor automatizada apunta a explorar la disfuncionalidad del lenguaje. Los movimientos compulsivos buscan generar experiencias limitrofes entre cuerpo y lenguaje, entre el "gesto humano y el funcionamiento mecánico de los engranajes" ${ }^{28}$. Allí el sonido desempeña un papel preponderante. En efecto, su primera exposición en Galería Central (San Pablo), llevada a cabo entre abril y mayo de 2017 bajo la curaduría de Juliana Gontijo, fue titulada Rrrrrrrrr. El nombre de la muestra, integrada por Máquina \#4 entre otras instalaciones, condensaba la onomatopeya del ruido de toda máquina, independientemente del trabajo específico que el artefacto efectúe. De acuerdo con la perspectiva de Gontijo, ante el "imperativo de precisión y rigor tecnocrático" ${ }^{29}$, un conjunto de prácticas artísticas contemporáneas buscan reelaborar poéticamente los materiales desde un enfoque crítico que subvierte la concepción utilitaria de los objetos, basada en la noción de progreso técnico. Mientras que en Máquina \#4 la piedra no era agujereada, sino tal vez apenas marcada como consecuencia del golpe obstinado del dispositivo, otras obras de Mureb sí dejaban marcas explícitas en la sala de exhibición a lo largo de su acción performática. Este es el caso de Máquina \#2: péndulo (2012), donde una vara metálica sujetada a la pared remata en un bloque de cemento. El movimiento pendular de la estructura hace que el monolito se desplace continuamente de izquierda a derecha y viceversa, dejando impreso sobre el muro dos líneas negras, a modo de surcos o trazos resultantes del vaivén repetido.

La falibilidad de la máquina también ha sido el núcleo central de la pesquisa del artista chileno Daniel Reyes León. En la instalación Una tonelada de lluvia (2016), desarrollada en el marco del Laboratorio Arte y $\mathrm{Crisis}^{30}$, un

28 GONTIJO, Juliana, "Leimotiv para Rrrrrrrrr", 2017, s/p. Disponible en: https://juligontijo.wordpress. com/2017/04/10/rrrrrrrrrr-exposicao-de-gabriela-mureb/ [consulta: 18-11-19].

29 GONTIJO, Juliana, Distopias Tecnológicas, Río de Janeiro, Circuito, 2014, p. 29.

30 El Laboratorio Arte y Crisis fue ideado por el centro interdisciplinario de investigación Núcleo Milenio Mo- sistema de refrigeración recubierto de hielo, dispuesto perpendicularmente con respecto al suelo de la sala, poco a poco condensa el agua. En la medida en que el hielo se derrite, sus gotas caen sobre un espejo de agua ubicado sobre el suelo. Interpretado como una metáfora de la frialdad de los sistemas sociales, cuyo funcionamiento en ocasiones pareciera desconocer la realidad de su entorno ${ }^{31}$, el mecanismo ideado por Reyes evidencia y cuestiona las estructuras naturalizadas que operan de manera inadvertida en el imaginario colectivo. Así, la falla del aparato permite entrever aquellas lógicas que suelen permanecer ocultas. Pero a juicio de Carol Illanes, aunque la obra de Reyes haya sido vinculada con el tópico del error de la máquina, en ella “(...) la falla esperada, pronosticada, se vuelve entonces una expectativa" ${ }^{32}$. Por lo tanto, si el espectador es invitado a contemplar la conducta incesante de la debacle, en realidad no es enfrentado al colapso del sistema, sino a su "funcionamiento sostenido" ${ }^{33}$. En este sentido, Illanes encuentra una relación entre el rol de las máquinas y la función de las ruinas atribuida por Marc Augé en su obra Tiempo en ruinas: evitar que el paisaje se diluya en una naturaleza inhabitada por el hombre, en la cual no exista la dimensión del tiempo histórico. De allí que Una tonelada de lluvia pueda ser leída en tanto "ruina musealizada del futuro" ${ }^{34}$.

delos de Crisis, y llevado a cabo en Chile durante el 2016. A lo largo del año, nueve artistas tuvieron la posibilidad de dialogar con un conjunto de científicos y reflexionar en conjunto sobre las formas de conocimientos para abordar las crisis sociales. Las obras resultantes de este proceso de trabajo fueron exhibidas en la exposición Crisis, Crisis, Crisis: La normalidad de la emergencia, curada por Montserrat Rojas en el Centro Cultural Matucana 100, presentada entre el 16 de noviembre de 2016 y el 6 de enero de 2017. Los artistas que participaron fueron Sebastián Calfuqueo, Gonzalo Cueto, Roberto Garretón, Nicolás Grum, Cristián Inostroza, Carolina Pino, Soledad Pinto, Daniel Reyes León y Pablo Rivera.

31 CORDERO, Rodrigo y MASCAREÑO, Aldo, Estéticas de la crisis. Modelos de mundos posibles, Santiago de Chile, Recrea, 2017, p.19.

32 ILLANES, Carol, "Desde el museo del futuro. Sobre el proyecto Una tonelada de lluvia de Daniel Reyes León" (Manuscrito), Santiago de Chile, 2017, p. 2.

33 ILLANES, Carol, "Desde el museo del futuro...", opus cit., p. 2.

34 ILLANES, Carol, "Desde el museo del futuro...", opus cit., p. 3. 


\section{Consideraciones finales}

En los proyectos examinados a lo largo de estas páginas, diversos medios y herramientas desencadenan actos de devastación, investigan las producción de vestigios en el transcurso del tiempo, e/o indagan las posibilidades de transformar restos de acciones transcurridas en el pasado en nuevas materialidades.

En este punto, cabe inquirir: ¿Cuál es el diferencial que introduce la destrucción en el ámbito de las artes tecnológicas con respecto al arte contemporáneo ajeno a la experimentación con las tecnologías? ¿Cómo opera la destrucción en aquellas obras en las cuales el cataclismo no es representado, simulado ni capturado de manera estática, sino que efectivamente, al igual que en Hommage à New York, acontece durante la exhibición de la pieza? ¿Cuáles son las consecuencias de la inclusión del componente performático de la destrucción? Postulamos que estas búsquedas y estrategias contemporáneas pueden ser leídas como "tácticas de destrucción constructiva": los elementos que confluyen en los trabajos tienden a perecer en el tiempo son destruidos, deteriorados o transfigurados-, hackeando la supuesta eficiencia e infalibilidad de la máquina. No obstante, sus vestigios no remiten a un pasado perdido, sino que se reconfiguran creativamente en el presente a partir de los propios comportamientos comprometidos por las obras. De ese modo, ponen en práctica una memoria actuante que constituye una modulación específica de las relaciones establecidas entre arte, tecnología y memoria.

Ahora bien, las implicancias estéticas, poéticas y conceptuales de la devastación y la catástrofe en el arte contemporáneo no deberían ser pensadas como un fenómeno totalizante, homogéneo e indiscriminado, sino leídas en función de las características específicas de los respectivos contextos de investigación y producción. Así como la propuesta de Gustav Metzger supuso una exploración doblemente estética y política, inseparable de la coyuntura de la segunda posguerra, las obras relevadas vibran, tiemblan, golpean, desbordan y se derriten en un ámbito convulsionado como el latinoamericano, donde históricamente la inestabilidad social, económica y política ha sido una circunstancia recurrente. Los proyectos que hemos analizado demuestran que el acto destructivo puede efectivamente encarnar una potente operación crítica en el arte latinoamericano a la hora de elucidar los modos en que la región se encuentra al borde del colapso inminente. Desde esta perspectiva, además de entrañar tácticas de destrucción constructiva, las obras estudiadas son concebidas como tácticas de transformación y resistencia que conducen en una dimensión tan simbólica como literal- a sacudir, agitar e inquietar al status quo. 